Journal of Animal and Veterinary Advances 11 (16): 2957-2963, 2012

ISSN: $1680-5593$

(C) Medwell Journals, 2012

\title{
The Nucleocapsid Protein of Avian Infectious Bronchitis Virus Interacts with Chicken Ubiquitin-Conjugating Enzyme 9 (cUBC9)
}

\author{
Sujun Zhao, Hongning Wang, Wenqiao Fan, Xin Yang, Anyun Zhang, Bo Zeng and Yi Zhang \\ Animal Disease Prevention and Food Safety Key Laboratory of Sichuan Province, \\ Key Laboratory of Bio-Resources and Eco-Environment of Ministry of Education, \\ School of Life Science, 985 Project Science Innovative Platform for Resource and \\ Environment Protection of Southwestern, Sichuan University, 610064 Chengdu, China
}

\begin{abstract}
Avian Infectious Bronchitis Virus (IBV) is an avian coronavirus. The Nucleocapsid (N) protein of IBV (IBV-N) is an important structural and functional protein and plays an essential role throughout the virus life cycle. Some cellular proteins may be involved in these complicated processes, little is known about virus host interactions. In this study, host cell proteins that interact with $\mathrm{N}$ protein were sought by screening a chicken lung cDNA library using a yeast two-hybrid system assay. IBV-N was shown to interact specifically with chicken Ubiquitin-Conjugating enzyme 9 (cUBC9) which belongs to the ubiquitin-conjugating enzyme family and is an important enzyme in the sumoylation system. Furthermore, the interaction between IBV-N and cUBC9 was confirmed by coimmunoprecipitation. The results of mapping the cUBC9 binding region of IBV-N indicated that N151-230 fragment of IBV-N is responsible for the majority of the binding to cUBC9. This virus-host protein interaction may provide insights into the function of IBV-N and facilitate rational targeting of the viral nucleocapsid protein for the development of structure-based vaccines and antiviral compounds.
\end{abstract}

\section{Key words: IBV, nucleocapsid protein, cUBC9, interaction, yeast two-hybrid, coimmunoprecipitation}

\section{INTRODUCTION}

Infectious Bronchitis Virus (IBV) causes an acute and contagious disease in domestic fowl with a significant impact on the poultry industry worldwide. It can replicate at many epithelial surfaces of the respiratory tract, alimentary tract, oviduct and kidney (Cavanagh, 2007). IBV is an enveloped positive-stranded RNA virus belonging to the family Coronaviridae and contains a genome of approximately $27 \mathrm{~kb}$ in length (Boursnell et al., 1987) that encodes four major structural proteins including the Spike glycoprotein (S), Nucleocapsid protein $(\mathrm{N})$, Membrane protein $(\mathrm{M})$ and Envelope protein (E). The N protein of IBV (IBV-N), a basic, phosphorylated structural protein of 409 residues is highly conserved among IBV strains, especially within the middle region (Spencer et al., 2008).

Earlier studies have indicated that IBV-N plays an essential role in genome replication, transcription of subgenomic RNA, translation and formation of the nucleocapsid (Dove et al., 2006; Schelle et al., 2005; Zhao et al., 2004). However, details about the possible involvement of $\mathrm{N}$ protein in IBV pathogenesis are still unclear. The coronaviruses $\mathrm{N}$ protein binds viral RNA to form a helical Ribonucleoprotein (RNP) that comprises the viral core structure (Chang et al., 2009; Hurst et al., 2005;
Ma et al., 2010; Saikatendu et al., 2007; Zhao et al., 2004), one of the key processes in the assembly of the virion and is essential for viability.

The $\mathrm{N}$ protein is the most abundant viral protein in coronaviruses and is produced throughout infection. Several functions have been postulated for the coronavirus $\mathrm{N}$ protein throughout the virus life cycle including viral packaging, viral core formation and signal transduction (Chang et al., 2009; Chen et al., 2009). It colocalizes with the replication complex in the sites of RNA synthesis (Hurst et al., 2005; Verheije et al., 2010). Inaddition, it has been shown that coronavirus $\mathrm{N}$ proteins may have RNA chaperone activity, binding RNA nonspecifically (Enjuanes et al., 2006; Zuniga et al., 2007). The RNA-binding (Fan et al., 2005; Grossoehme et al., 2009) and the oligomerization domains (Fan et al., 2005; Hurst et al., 2010) have been mapped. In particular, a serine-rich motif is probably important for the biological function of $\mathrm{N}$ protein and is conserved in all coronavirus $\mathrm{N}$ proteins (Tylor et al., 2009). Many studies have indicated that coronavirus $\mathrm{N}$ protein enters the host cell with the viral RNA and interferes with several cellular processes including induction of apoptosis and cell cycle arrest (Enjuanes et al., 2006) some of which involve interactions between $\mathrm{N}$ protein and host cell proteins (Surjit and Lal, 2008; Surjit et al., 2006) and may play a role in the pathology of coronavirus infections.

Corresponding Author: Hongning Wang, School of Life Science, Sichuan University, Wangjiang Road, Chengdu, P.R. China 
A yeast two-hybrid screen system was used to search for host cell proteins that may interact with IBV N protein in this study and it is demonstrated that the IBV $\mathrm{N}$ protein speciacally interacts with chicken UbiquitinConjugating enzyme 9 (cUBC9). cUBC9 belongs to the ubiquitin- conjugating enzyme family is involved in the process of protein sumoylation and is an important enzyme in the sumoylation system. This virus-host protein interaction may provide insights into understand better how IBV exploits its host.

\section{MATERIALS AND METHODS}

Virus and cell line: A nephro-pathogenic strain of IBV SAIBk was isolated from a field outbreak in Sichuan province in China. The SAIBK strain was propagated in the allantoic cavities of 10 days old SPF embryonated chicken eggs and the allantoic fluid was collected for viral isolation. The $50 \%$ chicken Infectious Dose $\left(\mathrm{EID}_{50}\right)$ was determined by inoculation serial 10 fold dilutions of virus into 10 days old SPF embryonated chicken eggs. Human embryonic kidney 293T cells (HEK 293T cells) were maintained in Dulbecco's Modified Eagle's Medium (DMEM) containing 10\% (vol/vol) Fetal Bovine Serum (FBS) and cultured in $5 \% \mathrm{CO}_{2}$ at $37^{\circ} \mathrm{C}$. Transient transfection assays were performed using Lipofectamine 2000 (Invitrogen, Carlsbad, CA) according to the manufacturer's protocol.

Plasmids and construction of recombinant vectors: The coding region of the $I B V-N$ gene and the truncated mutants N1-150, N151-230, N231-330 and N331-409 were subcloned into the yeast two-hybrid vector pGBKT7. A cDNA library of chicken lung mRNA was used for screening. The cDNA library and SmaIlinearised pGADT7-Rec were cotransformed into yeast strain AH109. The $c U B C 9$ gene was amplified by PCR from the chicken lung cDNA library. For expression in mammalian cells, the IBV-N and $c U B C 9$ genes were cloned into PCMV-HA and PCMV-Myc, respectively with corresponding restriction enzyme cleavage sites. All of the vectors were purchased from American Clontech.

Yeast two-hybrid assays: The Matchmaker Library Construction and Screening kit (Clontech) was used to test the interactions between IBV-N and host cell proteins in vitro. The chicken lung cDNA library in the yeast plasmid pGADT7-Rec vector was screened in the yeast strain AH109. All procedures including incubation of the yeast, construction of a DNA-BD fusion vector, generation of the cDNA library, transformation, mating and screening followed the protocols provided by the manufacturer. To identify positive interactions, the phenotypes of positive colonies were retested by restreaking on SD dropout plates 2-3 times and tested in a $\beta$-galactosidase activity assay as described in the users' manual. The cDNA inserts were rescued by plasmid isolation or PCR colony screening followed by sequencing. Finally, the interactions were retested in yeast by either cotransformation or mating.

Coimmunoprecipitation and immunoblot assay: To confirm the results observed with the yeast twohybrid system, another independent assay and coimmunoprecipitation was carried out. HEK 293T cells were transiently co-transfected with pCMV-HA-N and pCMV-Myc-cUBC9. At $48 \mathrm{~h}$ posttransfection, the cells were harvested and washed with Phosphate-Buffered Saline (PBS) then lysed using cold mild lysis buffer. Cell lysates were clarified by centrifugation at $12,000 \mathrm{~g}$ for $20 \mathrm{~min}$ at $4^{\circ} \mathrm{C}$ and the supernatants were then immunoprecipitated with primary antibody bound to protein A/G-Sepharose beads (Oncogene, San Diego, CA, USA) and incubated overnight with shaking.

The beads were collected by centrifugation and washed five times with wash buffer. Eluted proteins were resuspended in $2 \times$ Sodium Dodecyl Sulfate (SDS) sample buffer and denatured for $5 \mathrm{~min}$ in boiling water before SDS-Polyacrylamide Gel Electrophoresis (PAGE) for protein separation then finally analyzed by Western blotting. For Western blotting, samples obtained from coimmunoprecipitation assays or transfected cell lysates were separated by $12 \%$ SDS-PAGE and transferred onto polyvinylidene diflouride membranes. The membranes were then incubated with anti-HA (HA-tag polyclonal antibody, Clontech) or anti-c-Myc (c-Myc monoclonal antibody, Clontech) antibody followed by horseradish peroxidase-conjugated rabbit anti-goat or anti-mouse antibodies, respectively. Binding was visualized by enhanced chemiluminescence.

\section{RESULTS AND DISCUSSION}

\section{Identification of cUBC9 as a novel IBV-N interacting} protein using the yeast two-hybrid system: The $3 \times 10^{5}$ transformants were screened by mating and a total of 500 colonies grew up on Quadruple Drop-out (Leu-/Trp/Ade-/His-) selection plates. Upon further screening, 26 positive pGADT7-cDNA plasmids were successfully isolated from yeast cells and rescued in $E$. coli. The cDNA insert from each plasmid was amplified by PCR with vector primers and digested with HaeIII and Sau3AI to distinguish different fragments.

The results of cotransformation of yeast $\mathrm{AH} 109$ with pGBKT7-N and positive pGADT7-cDNA plasmids as well as positive and negative controls. The $\beta$-galactosidase 
activity of these clones was also assessed. In the filter assay the positiv e transformants were blue whereas false positives remain colorless or were a very light color (Fig. 1a). There were 9 confirmed positive clones and following sequence analysis showed that four were identified as chicken ubiquitin-conjugating enzyme 9 (cUBC9). As shown in Fig. 1b, the protein encoded by the pGADT7-cUBC9 clones interacted specifically with the IBV-N protein.
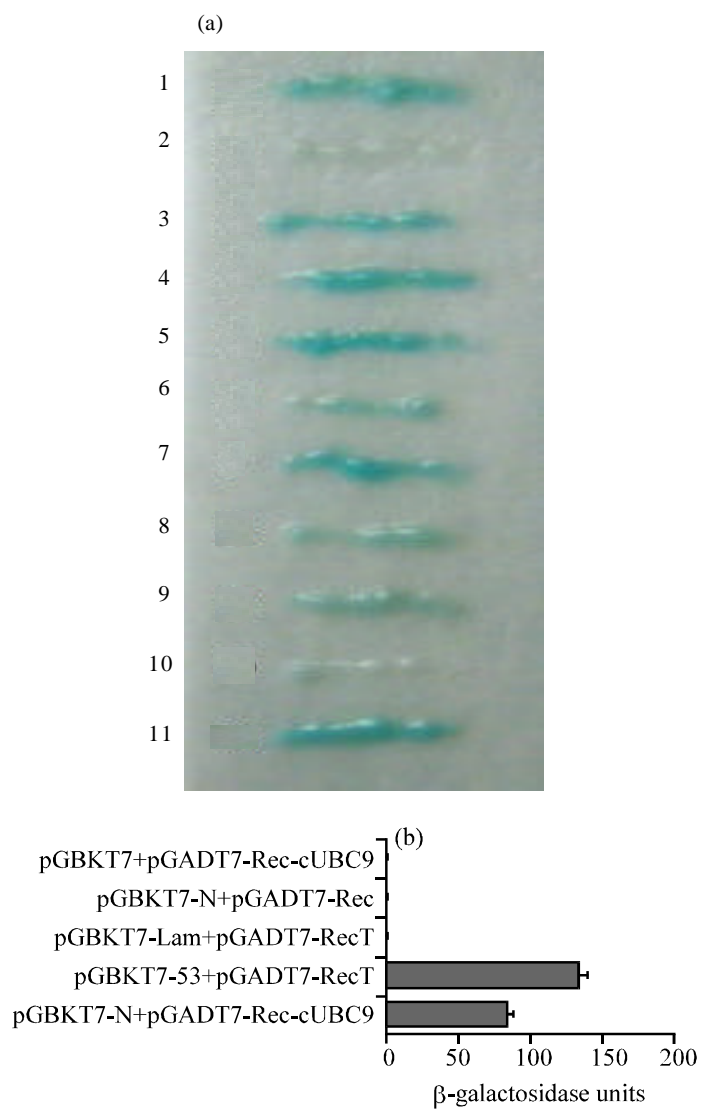

Fig. 1: $\beta$-galactosidase assay in yeast two-hybrid analysis showing positive and negative results. a) $\beta$ galactosidase filter assay. 1: Positive control. 2: Negative control. 3-11: eight randomly chose clones. 3, 4, 5, 7, 11 showing strong positive, in contrast, 6, 8, 9, 10 showing negative. b) Quantitation assay of $\beta$-examining the interaction between IBV-N and cUBC9. The plasmid pairs above were cotransfected into yeast strain AH1 09 and assessed for $\beta$-galactosidase activity. Results shown are the average units for triplicate assays. Error bars represent standard deviation

Cloning and sequence analysis of $\mathrm{BBV}-\mathrm{N}$ and cUBC9: The cUBC9 gene was cloned after PCR amplification from the chicken lung cDNA library. The Open Reading Frame (ORF) of cUBC9 is $477 \mathrm{bp}$ and encodes a polypeptide of 158 amino acids. The coding region of the $I B V-N$ gene was amplified by PCR from pMD18-T-N.

The amino acid sequence of cUBC9 was aligned with 4 known sequences of UBC9 proteins in GenBank including those of humans, Mus musculus, Drosophila melanogaster and Saccharomyces cerevisiaes (Fig. 2). The cUBC9 proteins were highly conserved, especially in higher eukaryotes which had identical amino acid sequences. The amino acid sequence of cUBC9 had about $85 \%$ identity with UBC9 of Drosophila melanogaster and only $56 \%$ identity with UBC9 of Saccharomyces cerevisiae. UBC9 is an important enzyme in the sumoylation system and many studies have indicated that the enzymatic active site is $\mathrm{C} 93$ amino acid residue (Duan et al., 2009; Fan et al., 2006) which is conserved in eukaryote (Fig. 2).

IBV-N has a lysine-rich region which has been predicted to be the Nuclear Localization Signal (NLS) in the C-terminal domain and a SR-rich region has been identified in the central domain that may be involved in RNA binding and protein-protein interactions. The results are shown in Fig. 3.

IBV-N physically associates with cUBC9 was revealed by coimmunoprecipitation: The results of immunoblotting assay showed that similar amounts of HA-tagged IBV-N protein and c-Myc-tagged cUBC9 protein were expressed when cells were transfected with pCMV-HA-N and pCMV-Myc-cUBC9, either individually (Fig. 4a, Lanes 1 and 2 ) or in combination (Fig. 4a, Lane 3) which were detected by their corresponding antibody. The results of coimmunoprecipitation were shown in Fig. 4b. IBV-N fusion protein was detected by Immunoprecipitation (IP) using anti-c-Myc antibody and Western blot using antiHA antibody (Fig. 4b, Lane 3). In addition, the cUBC9 fusion protein was detected by Immunoprecipitation (IP) using anti-HA antibody and Western blot using anti-cMyc antibody (Fig. 4b, Lane 3). The specificity of IBV-N binding to $\mathrm{cUBC} 9$ was tested by cotransfection of the empty Myc vector with pCMV-HA-N and the empty HA vector with pCMV-Myc-cUBC9 (Fig. 4b, Lanes 1 and 2).

\section{Mapping the cUBC9 binding region of $\mathrm{BBV}-\mathrm{N}$ using the} yeast two-hybrid system: These four truncated $\mathrm{N}$ proteins were tested using the two-hybrid assay in yeast (Fig. 5b). The results indicated that the $\beta$-galactosidase activity of N151-230 fragment containing the SR-rich motif and $\mathrm{AK}^{218} \mathrm{AE}$ is much higher than the activity of other 


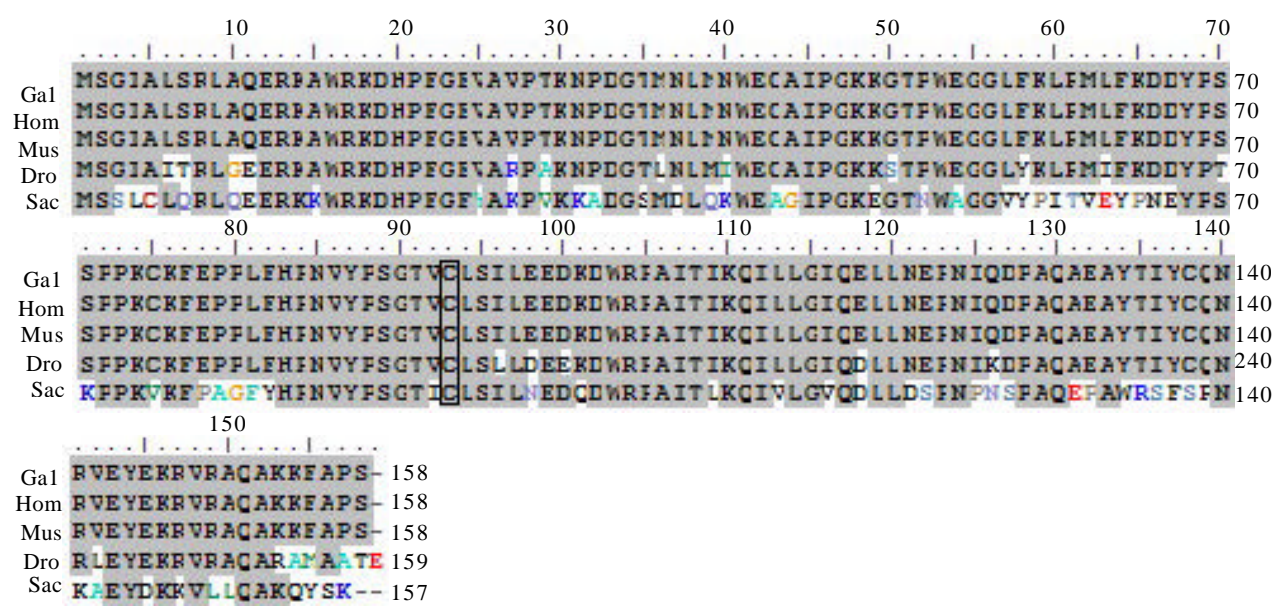

Fig. 2: The multiple alignments of UBC9 constructed by Clustal W. The source of the sequence: Chi-chicken GI: 19110799; Hom-Homo sapiens GI: 2597930; Mus-Mus musculus GI: 1943758; Dro-Drosophila melanogaster GI: 3892911; Sac-Saccharomyces cerevisiae GI: 1431070. Conserved residues are highlighted in gray. The enzyme active site $\mathrm{C} 93$ is conserved in the UBC9 which is boxed

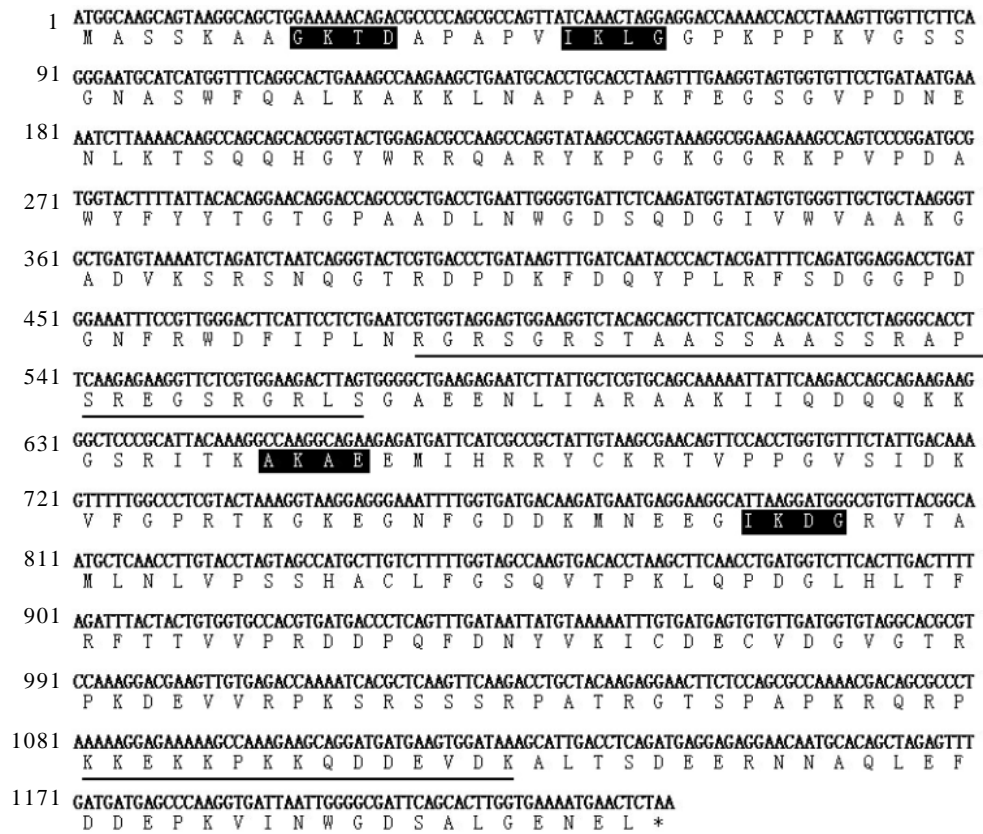

Fig. 3: Nucleic acid and amino acid sequences of IBV-N. Asterisk shows the termination codon. The sequences of SR-rich region and lysine-rich region are underlined and the consensus $\psi \mathrm{KXE}$ motif for sumoylation (where $\psi$ is usually a hydrophobic residue and $\mathrm{X}$ is any amino acid) are marked with shaded background

fragments and which is responsible for the majority of the binding to cUBC9. Using the yeast two-hybrid screen, cUBC9 was identified as a potential host cell target of IBV-N. During viral infection many viral proteins are post-translationally modified by viral or cellular enzymes (Ahn et al., 2001; Viswanathan et al., 2010). Sumoylation is a post-translational modification that is involved in the regulation of proteins of different cellular processes (Niedenthal, 2007). It is the process of covalent modification of cellular proteins by SUMO. UBC9 is an essential enzyme in the SUMO protein modification system and covalently conjugates SUMO1 to target proteins and modulates various cellular processes. 
(a)

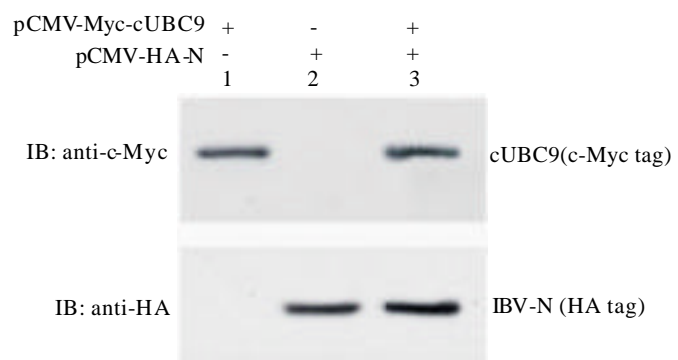

(b)

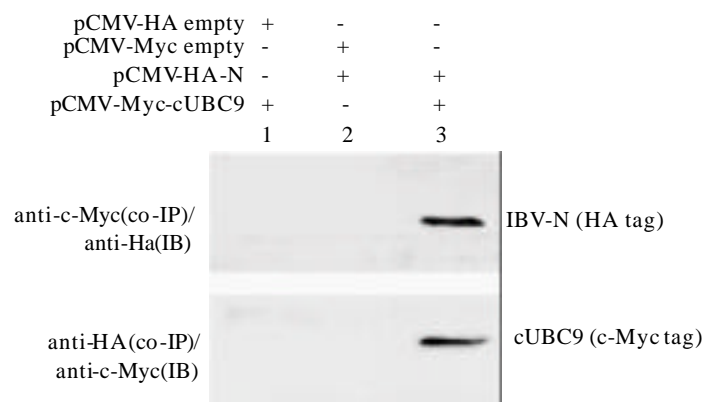

Fig. 4: Coimmunoprecipitation experiments for IBV-N and cUBC9 interaction; a) Immunoblotting; b) for IBV-N and cUBC9 were detected using anti-HA and anti-c-Myc, respectively to confirm the expression of the interested proteins. HA-tagged IBV-N protein and c-Myc-tagged cUBC9 protein were expressed respectively in HEK 293T cells. The plasmids used in lanes 1-3 were marked by plus and minus sign in the top; b) In coimmunoprecipitation (co-IP) for IBV-N and cUBC9, anti-c-Myc (or anti-HA) antibody was used for Immunoprecipitation (IP) and anti-HA (or anti-c-Myc) antibody was used for detection. Different combinations of the plasmids which were marked by plus and minus sign in the top of lanes 1-3 were used for cotransfection

The study suggests that sumoylation of IBV-N may occur. Earlier studies have shown that sumoylation modulates protein function through post-translational covalent attachment to lysine residues within targeted proteins (Johnson, 2004; Mo and Moschos, 2005; Muller et al., 2004). A tetrapeptide $\psi \mathrm{KXE}$ (where $\psi$ is usually a hydrophobic residue and $\mathrm{X}$ is any amino acid) has been defined as the consensus motif for sumoylation of target proteins containing an acceptor lysine residue (Seeler and Dejean, 2003). The nucleocapsid protein of some viruses can be sumoylated at this consensus acceptor lysine (Lee et al., 2003; Maeda et al., 2003). (a)

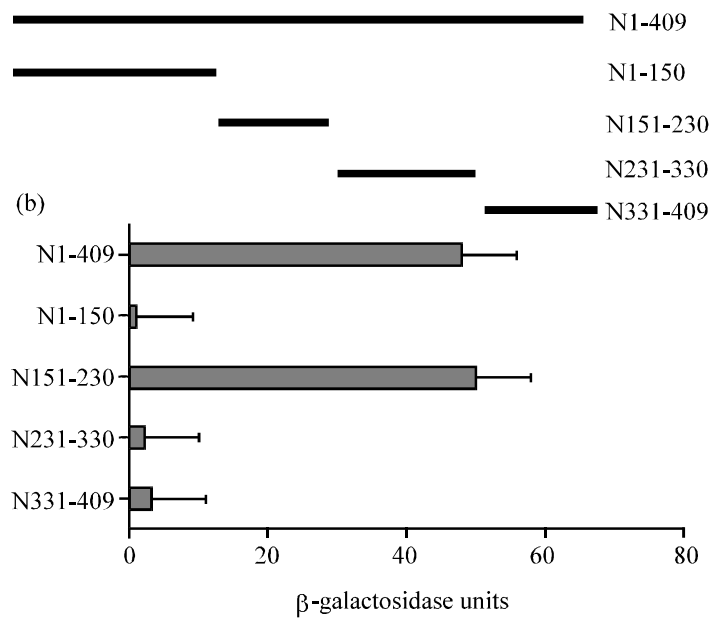

Fig. 5: Mapping the interaction domain of the IBV N protein; a) Schematic representation of the IBV N protein and the truncated mutants used in the yeast two-hybrid system; b) Quantitation assay of $\beta$-galactosidase activity was used to assess the binding ability of the truncated $\mathrm{N}$ protein mutants. The fragment N151-230 showed intensive binding to cUBC9. Results shown are the average units for triplicate assays. Error bars represent standard deviation

Researchers found three motifs, $\mathbb{K}^{18} \mathrm{LG}, \mathrm{AK}^{218} \mathrm{AE}$ and $\mathrm{IK}^{264} \mathrm{DG}$ which are the consensus motif for sumoylation in the $\mathrm{N}$ protein (Fig. 3). In this study, the results showed that $\mathrm{AK}^{218} \mathrm{AE}$ may be responsible for the majority of the binding to cUBC9. But studies on SARS-CoV have indicated that the consensus motif for sumoylation, $\mathrm{GK}^{62} \mathrm{EE}$ in the $\mathrm{N}$ protein is not responsible for binding to hUBC9 (Fan et al., 2006).

The RNA binding region in coronavirus $\mathrm{N}$ proteins overlaps with the binding site of UBC9 (Xu et al., 2002). The NTD and CTD of IBV-N, encompassing residues 1-171 and 268-407, respectively interact with the noncoding region of the viral genomic RNA at its 3 ' end (Zhou and Collisson, 2000). In this study, N151-230 fragment of IBV-N is responsible for the majority of the binding to $\mathrm{cUBC}$. Thus, the interaction with $\mathrm{CUBC} 9$ may affect the specificity of viral RNA binding by IBV-N and might regulate the activity and function of IBV-N influencing virus assembly. Thus, cUBC9 was identified as a novel host cell target of IBV-N in chicken and this interaction can be predicted to play a crucial role in the regulation of various cellular processes during viral infection. Further studies will be required to investigate these possibilities and gain a fuller understanding of the 
function of IBV-N and the potential pathogenic consequences of these virus-host protein interactions.

\section{CONCLUSION}

Researchers identified a novel host cell protein cUBC9 that interact specifically with $\mathrm{N}$ protein by screening a chicken lung cDNA library using a yeast two-hybrid system assay. cUBC9 belongs to the ubiquitin-conjugating enzyme family and is an important enzyme in the sumoylation system. Furthermore, the interaction between IBV-N and cUBC9 was confirmed by coimmunoprecipitation. Subsequently, the results of mapping the cUBC9 binding region of IBV-N indicated that N151-230 fragment of IBV-N containing the SR-rich motif and $\mathrm{AK}^{218} \mathrm{AE}$ is responsible for the majority of the binding to $\mathrm{CUBC}$. This virus-host protein interaction may provide insights into the function of IBV-N during viral infection and understand better how IBV exploits its host and facilitate rational targeting of the viral nucleocapsid protein for the development of structure-based vaccines and antiviral compounds.

\section{ACKNOWLEDGEMENTS}

This research was supported by the Foundation of Chinese National Programs for High Technology Research and Development (Project No.: 2006AA10A205, 2011AA10A209).

\section{REFERENCES}

Ahn, J.H., Y. Xu, W.J. Jang, M.J. Matunis and G.S. Hayward, 2001. Evaluation of interactions of human cytomegalovirus immediate-early IE2 regulatory protein with small ubiquitin-like modifiers and their conjugation enzyme Ubc9. J. Virol., 75: 3859-3872.

Boursnell, M.E.G., T.D.K. Brown, I.J. Foulds, P.F. Green, F.M. Tomley and M.M. Binns, 1987. Completion of the sequence of the genome of the Coronavirus avian infectious bronchitis virus. J. Gen. Virol., 68: 55-77.

Cavanagh, D., 2007. Coronavirus avian infectious bronchitis virus. Vet. Res., 38: 281-297.

Chang, C.K., Y.L. Hsu, Y.H. Chang, F.A. Chao and M.C. Wu et al., 2009. Multiple nucleic acid binding sites and intrinsic disorder of severe acute respiratory syndrome coronavirus nucleocapsid protein: Implications for ribonucleocapsid protein packaging. J. Virol., 83: 2255-2264.
Chen, C.Y., C.K. Chang, Y.W. Chang, S.C. Sue and H.I. Bai et al., 2009. Structure of the SARS coronavirus nucleocapsid protein RNA-binding dimerization domain suggests a mechanism for helical packaging of viral RNA. J. Mol. Biol., 368: 1075-1086.

Dove, B.K., J.H. You, M.L. Reed, S.R. Emmett, G. Brooks and J.A. Hiscox, 2006. Changes in nucleolar morphology and proteins during infection with the coronavirus infectious bronchitis virus. Cell Microbiol., 8: 1147-1157.

Duan, X., J.O. Trent and H. Ye, 2009. Targeting the SUMO E2 conjugating enzyme Ubc9 interaction for anticancer drug design. Anticancer Agents Med. Chem., 9: $51-54$.

Enjuanes, L., F. Almazan, I. Sola and S. Zuniga, 2006. Biochemical aspects of coronavirus replication and virus-host interaction. Annu. Rev. Microbiol., 60: $211-230$.

Fan, H., A. Ooi, Y.W. Tan, S. Wang, S. Fang, D.X. Liu and J. Lescar, 2005. The nucleocapsid protein of coronavirus infectious bronchitis virus: Crystal structure of its $\mathrm{N}$-terminal domain and multimerization properties. Structure, 13: 1859-1868.

Fan, Z., Y. Zhuo, X. Tan, Z. Zhou and J. Yuan et al., 2006. SARS-CoV nucleocapsid protein binds to hUbc9, a ubiquitin conjugating enzyme of the sumoylation system. J. Med. Virol., 78: 1365-1373.

Grossoehme, N.E., L. Li, S.C. Keane, P. Liu, C.E. 3rdDann, J.L. Leibowitz and D.P. Giedroc, 2009. Coronavirus N protein N-Terminal Domain (NTD) specifically binds the Transcriptional Regulatory Sequence (TRS) and melts TRS-cTRS RNA duplexes. J. Mol. Biol., 394: 544-557.

Hurst, K.R., L. Kuo, C.A. Koetzner, R. Ye, B. Hsue and P.S. Masters, 2005. A major determinant for membrane protein interaction localizes to the carboxy-terminal domain of the mouse coronavirus nucleocapsid protein. J. Virol., 79: 13285-13297.

Hurst, K.R., R. Ye, S.J. Goebel, P. Jayaraman and P.S. Masters, 2010. An interaction between the nucleocapsid protein and a component of the replicase-transcriptase complex is crucial for the infectivity of coronavirus genomic RNA. J. Virol., 84: 10276-10288.

Johnson, E.S., 2004. Protein modification by SUMO. Annu. Rev. Biochem., 73: 355-382.

Lee, B.H., K. Yoshimatsu, A. Maeda, K. Ochiai and M. Morimatsu et al., 2003. Association of the nucleocapsid protein of the Seoul and Hantaan hantaviruses with small ubiquitin-like modifier-1related molecules. Virus. Res., 98: 83-91. 
Ma, Y., X. Tong, X. Xu, X. Li, Z. Lou and Z. Rao, 2010. Structures of the N- and C-terminal domains of MHVA59 nucleocapsid protein corroborate a conserved RNA-protein binding mechanism in coronavirus. Protein Cell, 1: 688-697.

Maeda, A., B.H. Lee, K. Yoshimatsu, M. Saijo, I. Kurane, J. Arikawa and S. Morikawa, 2003. The intracellular association of the Nucleocapsid Protein (NP) of hantaan virus (HTNV) with small ubiquitin-like modifier-1 (SUMO-1) conjugating enzyme 9 (Ubc9). Virology, 305: 288-297.

Mo, Y.Y. and S.J. Moschos, 2005. Targeting Ubc9 for cancer therapy. Expert Opin Ther Targets, 9: 1203-1216.

Muller, S., A. Ledl and D. Schmidt, 2004. SUMO: A regulator of gene expression and genome integrity. Oncogene, 23: 1998-2008.

Niedenthal, R., 2007. Ubc9 Fusion-Directed SUMOylation (UFDS). Biochem. Soc. Trans., 35: 1430-1432.

Saikatendu, K.S., J.S. Joseph, V. Subramanian, B.W. Neuman, M.J. Buchmeier, R.C. Stevens and P. Kuhn, 2007. Ribonucleocapsid Formation of Severe Acute Respiratory Syndrome Coronavirus through Molecular Action of the N-Terminal Domain of $\mathrm{N}$ Protein. J. Virol., 81: 3913-3921.

Schelle, B., N. Karl, B. Ludewig, S.G. Siddell and V. Thiel, 2005. Selective Replication of Coronavirus Genomes That Express Nucleocapsid Protein. J. Virol., 79: 6620-6630.

Seeler, J.S. and A. Dejean, 2003. Nuclear and unclear functions of SUMO. Nat. Rev. Mol. Cell Biol., 4: 690-699.

Spencer, K.A., M. Dee, P. Britton and J.A. Hiscox, 2008. Role of phosphorylation clusters in the biology of the coronavirus infectious bronchitis virus nucleocapsid protein. Virology, 370: 373-381.

Surjit, M. and S.K. Lal, 2008. The SARS-CoV nucleocapsid protein: A protein with multifarious activities. Infect. Genet. Evol., 8: 397-405.
Surjit, M., B. Liu, V.T.K. Chow and S.K. Lal, 2006. The nucleocapsid protein of severe acute respiratory syndrome-coronavirus inhibits the activity of cyclincyclin-dependent kinase complex and blocks S phase progression in mammalian cells. J. Biol. Chem., 281: 10669-10681.

Tylor, S., A. Andonov, T. Cutts, J. Cao and E. Grudesky et al., 2009. The SR-rich motif in SARS-CoV nucleocapsid protein is important for virus replication. Can J. Microbiol., 55: 254-260.

Verheije, M.H., M.C. Hagemeijer, M. Ulasli, F. Reggiori, P.J. Rottier, P.S. Masters and C.A. De Haan, 2010. The coronavirus nucleocapsid protein is dynamically associated with the replication-transcription complexes. J. Virol., 84: 11575-11579.

Viswanathan, K., K. Fruh and V. DeFilippis, 2010. Viral hijacking of the host ubiquitin system to evade interferon responses. Curr. Opin Microbiol., 13: $517-523$.

Xu, X., W. Severson, N. Villegas, C.S. Schmaljohn and C.B. Jonsson, 2002. The RNA binding domain of the hantaan virus $\mathrm{N}$ protein maps to a central, conserved region. J. Virol., 76: 3301-3308.

Zhao, Y., S.W. Kwon, A. Anselmo, K. Kaur and M.A. White, 2004. Broad spectrum identification of cellular small ubiquitin-related modifier (SUMO) substrate proteins. J. Biol. Chem., 279: 20999-21002.

Zhou, M. and E.W. Collisson, 2000. The amino and carboxyl domains of the infectious bronchitis virus nucleocapsid protein interact with $3^{\prime}$ genomic RNA. Virus. Res., 67: 31-39.

Zuniga, S., I. Sola, J.L. Moreno, P. Sabella, J. Plana-Duran and L. Enjuanes, 2007. Coronavirus nucleocapsid protein is an RNA chaperone. Virol., 357: 215-227. 